\title{
A putative diacidic motif in the SARS-CoV ORF6 protein influences its subcellular localization and suppression of expression of co-transfected expression constructs
}

Vithiagaran Gunalan ${ }^{1,2,3}$, Ali Mirazimi ${ }^{1,2}$ and Yee-Joo $\operatorname{Tan}^{4^{*}}$

\begin{abstract}
Background: The ORF6 protein is one of the eight accessory proteins of the severe acute respiratory syndrome coronavirus (SARS-CoV). Numerous properties of ORF6 have been documented and this study focuses on two of these, namely, its ability to suppress the expression of co-transfected expression constructs and its subcellular localization to vesicular structures.

Results: Using a transient transfection system, ORF6's ability to suppress the expression of co-transfected expression constructs was measured in a quantitative manner. While ORF6 does not have a global effect on protein synthesis, quantitative real-time PCR revealed that it down-regulated the mRNA level of the co-transfected myc-nsp8 gene. Furthermore, alanine substitution of a diacidic cluster motif (aa53-56) in the ORF6 gene caused a reduction in the suppression of expression of co-transfected myc-nsp8 gene. Our previous study revealed that ORF6 localized to vesicular structures in SARS-CoV infected Vero E6 cells. Here, ORF6 was observed to be localized to similar vesicular structures in Vero E6 cells which have been transiently transfected with a mammalian expression plasmid encoding for untagged ORF6. ORF6 showed partial colocalization with cellular proteins CD63 and Lamp1, suggesting that the vesicular structures may be a subpopulation of endosomal/lysosomal vesicles. The alanine substitution of the diacidic cluster motif also altered the subcellular localization of the ORF6 protein, indicating a potential relationship between the subcellular localization of the ORF6 protein and its ability to suppress the expression of co-transfected expression constructs.

Conclusions: By combining quantitative real-time PCR and transient transfection system, a simple and safe method is established to measure ORF6's ability to suppress the expression of co-transfected myc-nsp8. In addition, immunofluorescence analysis revealed that the subcellular localization of ORF6 when expressed on its own is similar to that observed in SARS-CoV infected cells. Through the use of these two assays, a putative diacidic motif in the ORF6 protein was found to influence its subcellular localization and ability to suppress the expression of cotransfected expression constructs.
\end{abstract}

\section{Background}

An outbreak of Severe Acute Respiratory Syndrome (SARS) in 2003 which carried with it a fatality rate of $8 \%$ was traced to a novel coronavirus dubbed the SARS Coronavirus (SARS-CoV). This novel coronavirus was

\footnotetext{
* Correspondence: Yee_Joo_TAN@NUHS.edu.sg

${ }^{4}$ Department of Microbiology, Yong Loo Lin School of Medicine, National University Health System, National University of Singapore, MD4, 5 Science Drive 2, Singapore 117597, Singapore

Full list of author information is available at the end of the article
}

eventually classified as a Group IIb coronavirus, a subset of the Group II coronaviruses. The subclassification was, in part, due to the presence of several accessory genes in the coronavirus which have no known homologs within the family Coronaviridae. These accessory genes have been the subject of study by many groups (for reviews, see 1 and 2) and have been assigned a plethora of physical characteristics and intracellular functions. Most importantly, almost all of these accessory genes have been shown to be dispensable for viral replication 
in cell culture, with the exception of the 3a accessory gene (3). It has been suggested these accessory genes have subtle effects on SARS-CoV replication and may be more important for viral replication or pathogenesis in vivo.

One of these accessory genes, ORF6, encodes for a $\sim 7 \mathrm{kDa}$ protein with a hydrophobic $\mathrm{N}$-terminal and that has been suggested to have a N-endo-C-endo conformation (4). Several groups have undertaken to characterize the protein product of the ORF6 gene and found that it interacts with the nsp8 protein from the SARS replicase complex (5), is able to increase infection titer during early infection at low multiplicity of infection (6), increase the rate of cellular gene synthesis (7), inhibit interferon production (8), and inhibit the nuclear translocation of STAT1 by interacting with karyopherin $\alpha 2$ (9). Most recently, the ORF6 protein has been suggested to induce intracellular membrane rearrangements resulting in a vesicular population in the infected cell which could possibly serve some role in increasing replication (10). Such virus-induced or virus associated vesicles have previously been shown in other viral infections, such as protein trafficking in Herpes simplex virus (11) and Sendai virus (12). Members of the coronavirus family have been shown by several groups to also utilize vesicular structures within the infected cell; most of these studies suggest that vesicles play a role in viral replication (13-16).

We have previously shown that the ORF6 protein colocalizes with Lamp1-positive vesicles in SARS-CoV infection (5) and also with the nsp8 protein in the same set of infected cells, indicating a possible role for the ORF6 protein in the replicative process of SARS-CoV. However, there has been, to date, little work done to link the subcellular localization of the ORF6 protein to its known functions. Gallagher and co-workers have shown that the ability of ORF6 to impede nuclear translocation resulted in the suppression of expression of transgenes from co-transfected expression constructs if these need transcription in the nucleus (17). Using this knowledge, a simple co-transfection system and alanine substitution mutants in the ORF6 gene are used here to determine regions of the ORF6 protein that modulate its ability to suppress the expression of co-transfected myc-nsp8. The subcellular localization of these mutants is then examined by immunofluorescence experiments and compared to that of wildtype ORF6.

\section{Methods}

\section{Cells, Plasmids and Antibodies}

Vero E6 cells were obtained from ATCC, and cultured in DMEM (Invitrogen) supplemented with 10\% FBS (Gibco) and penicillin/streptomycin (Sigma). Cells were cultured in a $37^{\circ} \mathrm{C}$ incubator with $5 \% \mathrm{CO} 2$. ORF6 was cloned into the pXJ3'HA vector (T. Leung, Institute of
Molecular and Cell Biology, Singapore, personal communication) using the sites BamHI and XhoI with a stop codon to create pXJ3'-ORF6, expressing an untagged protein. The nsp8 gene from SARS-CoV was cloned using BamHI and XhoI sites into pXJ40myc to create pXJ40myc-nsp8, with an N-terminal myc tag for detection. GST was cloned into pXJ40flag to create pXJ40flag-GST, which expresses an N-terminal flag tagged GST protein. The ORF6A49-52 and ORF6A5356 mutants were generated by 2 rounds of PCR: the first round of PCR was used to generate 5' and 3' fragments of ORF6 containing the appropriate alanine substitutions; these were then amplified into full-length ORF6 using end primers for the ORF6 gene and cloned into pXJ3'HA using BamHI and XhoI sites. The following antibodies were used in this study: rabbit anti-ORF6 was purchased from Abgent, mouse anti-actin from Sigma, mouse anti-Lamp1 from Abcam, mouse anti-myc from Santa Cruz and mouse anti-CD63 was purchased from Biodesign International.

\section{Immunofluorescence}

Vero E6 cells were plated in $60 \mathrm{~mm}$ dishes containing coverslips and subsequently transfected with mammalian expression plasmids relevant to the experiment being performed. Trasfection was performed using Lipofectamine 2000 (Invitrogen) according the manufacturer's instructions. 16 hours after transfection, coverslips were prepared for immunofluorescence as follows: Fixation was carried out in $100 \%$ methanol for 5 minutes and coverslips were left to dry before being blocked with PBS containing 1\% BSA for 30 minutes. Primary antibody incubation was carried out in a humidified chamber for 1 hour before coverslips were washed 3 times in blocking solution. Secondary antibody incubation was then carried out for an hour in a humidified chamber before the coverslips were washed 3 times. Coverslips were mounted onto glass slides using a mounting mixture of $70 \%$ Fluorsave (Calbiochem) and 30\% Vectashield containing DAPI (Vector Laboratories). Slides were imaged using a Zeiss LSM510Meta confocal microscope (Zeiss).

\section{SARS-CoV Infection}

Vero E6 cells grown on coverslips were infected with SARS-CoV Frankfurt strain 1. When the cells showed $75 \%$ cytopathic effects, they were subjected to immunofluorescence as described above. All SARS-CoV infection work was carried out at the BSL4 facility at the Swedish Institute for Infectious Disease Control in Solna, Sweden.

\section{Western Blotting}

Vero E6 cells were transfected with relevant plasmids for each experiment. 16 hours after transfection, cells 
were scraped into media before being spun down in a benchtop centrifuge and washed twice with PBS. After washes, the cell pellets were resuspended in RIPA buffer (50 mM Tris (pH 8.0), $150 \mathrm{mM} \mathrm{NaCl}, 0.5 \%$ NP40, 0.5\% deoxycholic acid, $0.005 \%$ SDS) and freeze-thawed in liquid nitrogen 3 times before being spun down at 13K $\mathrm{rpm}$ to remove cellular debris. SDS loading buffer was added to clarified lysates before being boiled at $100^{\circ} \mathrm{C}$ and loaded onto an SDS-PAGE gel for separation. Gels containing separated lysates were transferred onto Hybond-C nitrocellulose membranes (Amersham) before blocking with $5 \%$ skimmed milk in PBS-Tween. Antibody incubations were carried out at $4^{\circ} \mathrm{C}$ overnight or at room temperature, with constant rotation. After each incubation, membranes were washed 3 times for 10 minutes each with PBS-Tween. Following washes after secondary antibody incubation, membranes were treated with chemiluminescent substrate (Thermo) and exposed to film.

\section{Metabolic labeling}

Vero E6 cells were transfected with $1 \mu \mathrm{g}$ or $2 \mathrm{ug}$ of either pXJ3'-ORF6 or empty vector. 16 hours post transfection, cells were starved using DMEM without L-glutamine, cysteine and methionine (Invitrogen) before being incubated with $100 \mu \mathrm{Ci}$ of ${ }^{35} \mathrm{~S}$-labelled cysteine and methionine (Perkin Elmer) diluted in starvation medium for 30 minutes. At the end of each incubation period, cells were washed in cold PBS and scraped into a centrifuge tube and lysed in RIPA buffer. $20 \mu \mathrm{g}$ of each lysed sample was boiled with SDS loading buffer and PAGE separated before being fixed and dried in a Model 583 Gel Dryer (Bio-Rad). Dried gels were exposed to film at $-80^{\circ} \mathrm{C}$ to obtain autoradiograph. Quantification of signals was performed using the gel analysis module in Image J.

\section{Quantitative Realtime PCR}

Vero E6 cells were co-transfected with increasing amounts of pXJ3'-ORF6 $(0-2 \mu \mathrm{g})$ and a fixed amount of pXJ40myc-nsp8 $(1 \mu \mathrm{g})$. For each sample, the total amount of DNA was normalized to $3 \mu \mathrm{g}$ by addition of the empty vector, pXJ3'HA, when necessary. 16 hours post-transfection, cells were scraped into media and spun down in a benchtop centrifuge. Cells were washed twice with PBS and cell pellets were resuspended with Trizol reagent (Invitrogen). Trizol samples were mixed with chloroform and spun down in a benchtop centrifuge at $4^{\circ} \mathrm{C}$ before being processed with an RNeasy Mini Kit (Qiagen), according to the manufacturer's instructions. RNA samples were quantified with a Nanodrop ND-1000 spectrophotometer (Nanodrop Technologies). Equal amounts of each RNA sample were then reverse transcribed using poly $\mathrm{d}(\mathrm{T})_{12-18}$ (Invitrogen) and with Superscript II reverse transcriptase (Invitrogen), following manufacturer instructions. Following reverse transcription, equal amounts of each cDNA sample were added to a portion of reaction mix (Roche) along with either of the following primer pairs: SARS-nsp8-F: 5'-TGAATGCTAAATCTGAGTTTGA-3', SARS-nsp8-R: 5'-CATAGCCTGATCTGCCATCTTTT-3', GAPDH-F: 5'-GAAGGTGAAGGTCGGAGTC-3', GAPDH-R: 5'GAAGATGGTGATGGGATTTC-3' and one of the following probes: SARS-nsp8-P: 5' 6-FAMCGTGATGCTGCCATGCAACGC-BHQ 3', GAPDH-P: 5' 6-FAM-CAAGCTTCCCGTTCTCAGCC-BHQ 3'. Reaction mixes were subjected to quantitative real-time PCR in a Roche Lightcycler 2.0. Cycle threshold values obtained for the SARS nsp 8 gene were normalized against those for GAPDH to obtain relative quotients which were then plotted for increasing amounts of pXJ3'-ORF6 transfected. Briefly, the Ct for the GAPDH control was subtracted from the $\mathrm{Ct}$ for the $n s p 8$ gene $(\Delta \mathrm{Ct})$. The fold changes were then calculated from the $\Delta \Delta \mathrm{Ct}$, which was obtained by subtracting each $\Delta \mathrm{Ct}$ from the $\Delta \mathrm{Ct}$ obtained when $2 \mu \mathrm{g}$ of $\mathrm{pXJ}$ 'HA vector was used. Alternatively, quantitative real-time PCR was carried out using SsoFast EvaGreen SYBR Green Master mix (Bio-Rad) using the same primers for $n s p 8$ and $G A P D H$ as listed above; probes were omitted from these experiments. These reactions were carried out in an ABI 7500Fast Realtime PCR system.

\section{Results}

The ORF6 protein exerts an effect on the expression of transgenes from co-transfected expression constructs

To determine amount of ORF6 required to exert an effect on the expression of transgenes from co-transfected expression constructs, Vero E6 cells were transfected with $1 \mu \mathrm{g}$ of $\mathrm{pXJmyc-nsp} 8$ alone or with either 1 $\mu \mathrm{g}, 2 \mu \mathrm{g}$ or $3 \mu \mathrm{g}$ of pXJ3'-ORF6 plasmid. Western blotting showed a dose-dependent reduction in the expression of nsp8, concomitant with an increase in the expression of ORF6 (Figure 1A). In order to determine the specificity of this effect, Vero E6 cells were cotransfected with another mammalian expression construct pXJflag-GST and pXJ3'-ORF6 in the same manner as previously done with the construct encoding for nsp8. When titrated against increasing amounts of ORF6 protein, the flag-tagged GST protein was also observed by Western Blotting to show reduced levels of expression in a dose-dependent manner (Figure 1B). Thus, it seems that ORF6 exerts an effect on the expression of transgenes from co-transfected expression constructs regardless of the nature of the transgenes. However, when increasing amounts of GST were titrated against nsp8 in the same manner, no significant reduction in nsp 8 expression was observed, indicating that this effect was specific to the ORF6 protein (Figure 1C). 
A

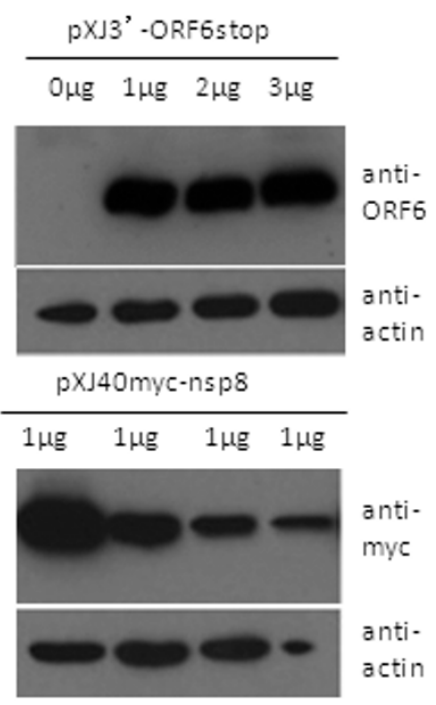

B

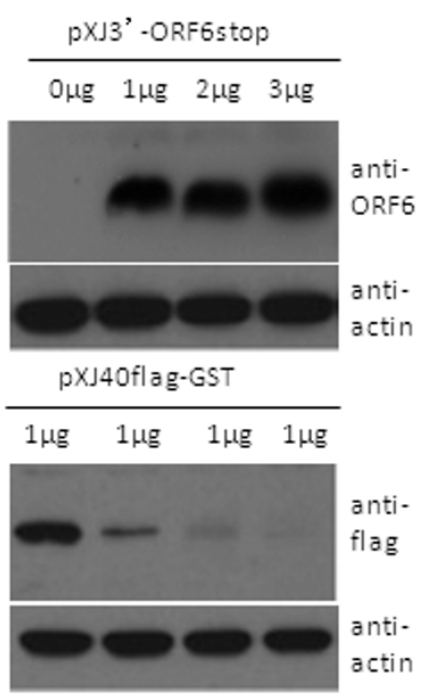

C

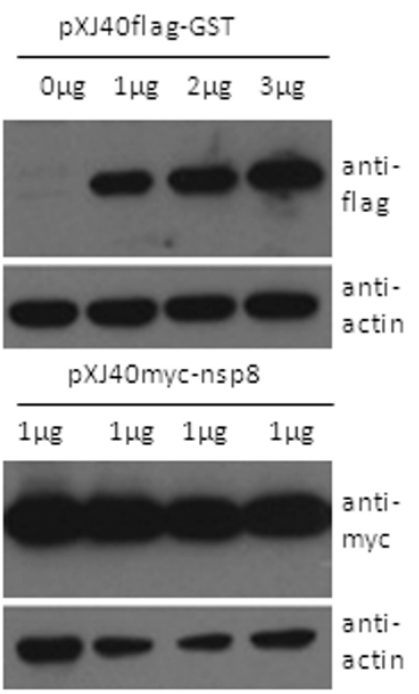

Figure 1 ORF6 specifically impedes expression of co-transfectants. Vero E6 cells were co-transfected with mammalian expression plasmids encoding for ORF6 and nsp8 (A), ORF6 and GST (B) or GST and nsp8 (C). Cells were lysed 16 hours post transfection and subjected to Western Blot analysis using equal amounts of lysate from each transfection. Blots were stripped post-detection and re-probed with an anti-actin antibody as a loading control. Similar results were obtained in three independent experiments, and a representative set of data is presented.

\section{ORF6 does not affect total cellular protein synthesis}

The ORF6 protein has been observed to cause a reduction in the expression of 2 different proteins, with different epitope tags. In order to examine the possibility that this downregulation could be a global effect, Vero E6 cells were transfected with either empty vector, pXJ3'HA, or pXJ3'-ORF6, and metabolically labeled with ${ }^{35} \mathrm{~S}$ for a period of 30 minutes. Quantification of the signals in each lane of the resulting autoradiograph was performed and the readings were normalized to the reading in the lane from the untransfected cells (Figure $2)$. When $1 \mu \mathrm{g}$ of DNA was used, there is a slight decrease in the total cellular protein synthesis in ORF6 expressing cells when compared to cells which had been transfected with empty vector. However, almost the same degrees of decrease in the total cellular protein synthesis were observed in both pXJ3'HA and pXJ3'ORF6 transfected cells when $2 \mu \mathrm{g}$ of DNA was used. Hence, the decrease in total cellular protein synthesis observed seems to be related to the transfection process rather than the expression of ORF6. This suggests that the cellular effect of ORF6 is not global.

\section{ORF6 exerts its effect at a transcriptional level}

In order to examine the possibility that ORF6 affects the expression of transgenes from co-transfected expression constructs via a transcriptional mechanism, Vero E6 cells were transfected with $1 \mu \mathrm{g}$ of pXJ40myc-nsp8 and either $1 \mu \mathrm{g}, 2 \mu \mathrm{g}$ of pXJ3'-ORF6 or no ORF6 plasmid.
16 hours post-transfection, total RNA was extracted, reverse transcribed and subjected to quantitative realtime PCR. Taqman chemistry was used to assay for $n s p 8$ and GAPDH was used as an endogenous control. The $\Delta \Delta \mathrm{Ct}$ method was used to calculate the nsp 8 mRNA level with respect to the level in the absence of ORF6. As shown in Figure 3, ORF6 caused a reduction in the level of nsp8 mRNA in a dose-dependent manner, indicating a transcriptional reduction caused by ORF6. These results suggest that ORF6 is able to exert some form of transcriptional inhibition, which is seen in the reduced expression from co-transfected plasmids.

\section{The ORF6 protein localizes to intracellular vesicles} positive for Lamp1 and CD63

It has been previously reported that the SARS-CoV ORF6 protein localizes to intracellular membranous compartments, which have been suggested to be induced by ORF6 itself (10). In agreement, it was observed in this study that ORF6 localized to vesicular compartments both in SARS-infected Vero E6 cells and Vero E6 cells transfected with a plasmid encoding for the ORF6 protein (Figure 4A). Vero E6 cells were infected with SARS-CoV and analyzed by immunofluoresence, using an antibody against the ORF6 protein. ORF6 was observed to localize to a distinct population of intracellular vesicles in these infected cells (Figure 4A). Following this, a mammalian expression plasmid pXJ3'-ORF6 was transfected into Vero E6 cells and 


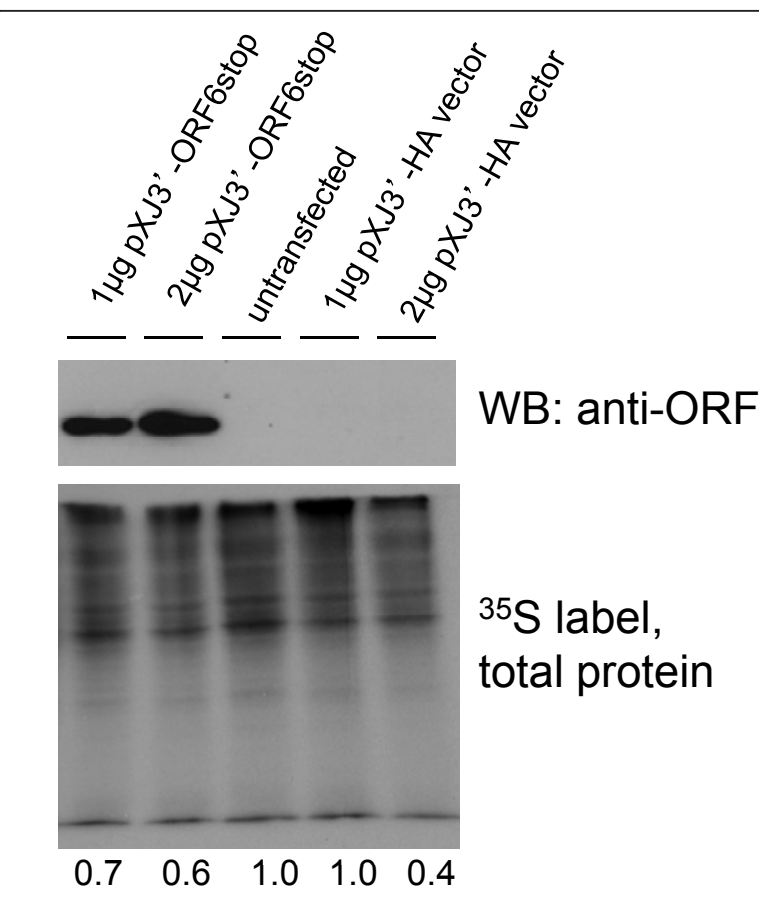

Figure 2 ORF6 expression in Vero E6 cells does not cause a reduction in total cellular protein. Vero E6 cells were transfected for 16 hours with increasing amounts of pXJ3'-ORF6 or an empty vector, pXJ3'HA. Cells were starved of methionine and cysteine for 1 hour and labeled with a mixture of 35S-labelled methionine and cysteine for 30 minutes, then subjected to SDS-PAGE separation and autoradiography (lower panel). Quantification of the signals in each lane of the resulting autoradiograph was performed and the readings, after normalization to the reading in the lane from the untransfected cells, are shown at the bottom of the autoradiograph. A fraction of lysate was subjected to Western Blotting using an antibody directed against the ORF6 protein (upper panel). Similar results were obtained in two independent experiments, and a representative set of data is presented.

analyzed in the same manner with the same antibody. Confocal microscopy showed that the ORF6 protein localized to a similar population of intracellular vesicles.

In our previous work studying the interaction between ORF6 and the nsp8 protein of SARS-CoV (5), we showed a colocalization of ORF6 and Lamp1 in Vero E6 cells infected with the HKU39849 strain of SARS-CoV. Similarly, there was a significant degree of colocalization between the Lamp1 and ORF6 in transiently transfected Vero E6 cells (Figure 4B), indicating that the ORF6positive vesicles seen in both infected cells and transiently transfected Vero E6 cells were probably an identical population of vesicles. This allowed us to then use pXJ3'-ORF6-transfected Vero E6 cells to further study the characteristics of this vesicular population. In addition to Lamp1, CD63 (a marker for late endosomes) was used to examine the compartmental characteristics of the vesicles. As shown in Figure 4B, ORF6-positive vesicles coincide significantly with CD63-positive vesicles in

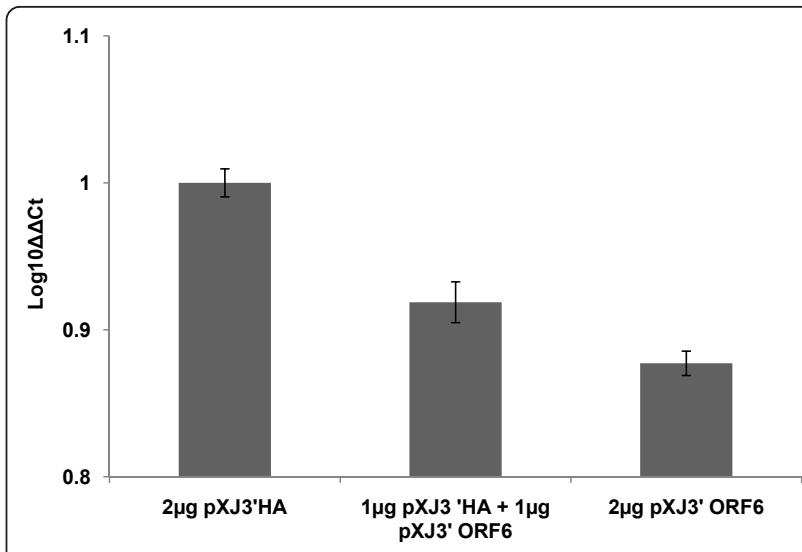

Figure 3 The ORF6 protein exerts a transcriptional effect on nsp8 expression. Vero E6 cells were co-transfected with $1 \mu \mathrm{g}$ of pXJ40myc-nsp8 and different amount of pXJ3'-ORF6. For each sample, the total amount of DNA was normalized to $3 \mu \mathrm{g}$ by addition of the empty vector, $\mathrm{PXJ} 3^{\prime} \mathrm{HA}$, when necessary. Total RNA was harvested 16 hours later, reverse transcribed and subjected to quantitative real-time Taqman PCR using primers and probes specific to nsp8 and cellular protein, GADPH. Relative quotients between the resulting $\mathrm{Ct}$ values for each sample were calculated using the $\Delta \Delta C t$ method. Similar results were obtained in three independent experiments, and a representative set of data is presented.

Vero E6 cells. This population of vesicles is therefore a subset of the late endosomal and lysosomal populations, and shows that the plasmid system employed here yields similar colocalization of ORF6 with cellular markers as seen in previous infection work (5).

\section{Amino Acids 53-56 in ORF6 constitute a putative diacidic motif which affects the suppression of the expression of co-transfected myc-nsp8}

Using the CBS Prediction Servers (http://www.cbs.dtu. $\mathrm{dk} /$ services/), it was determined that the ORF6 protein had several putative motifs of interest. Of these, aa49-52 (YSEL) was predicted to be a lysosomal targeting motif YXXL (18), and aa53-56 (DDEE) bears similarity to a putative diacidic motif $D x E$, which governs ER export and subsequent localization to different membranous compartments (19). These motifs were also predicted by Netland and co-workers (4). In order to determine the contribution of these motifs to the function of the ORF6 protein, alanine substitutions were introduced by two-step PCR to yield ORF6A49-52, which substituted four alanine residues for the YSEL region, and ORF6A53-56, which substituted four alanines for the DDEE region (Figure 5A).

These alanine substitution mutants were cloned into the same vector as the wildtype ORF6 gene and titrated against the $n s p 8$ gene, by co-transfection of Vero E6 cells with plasmids encoding for myc-nsp8 and either 


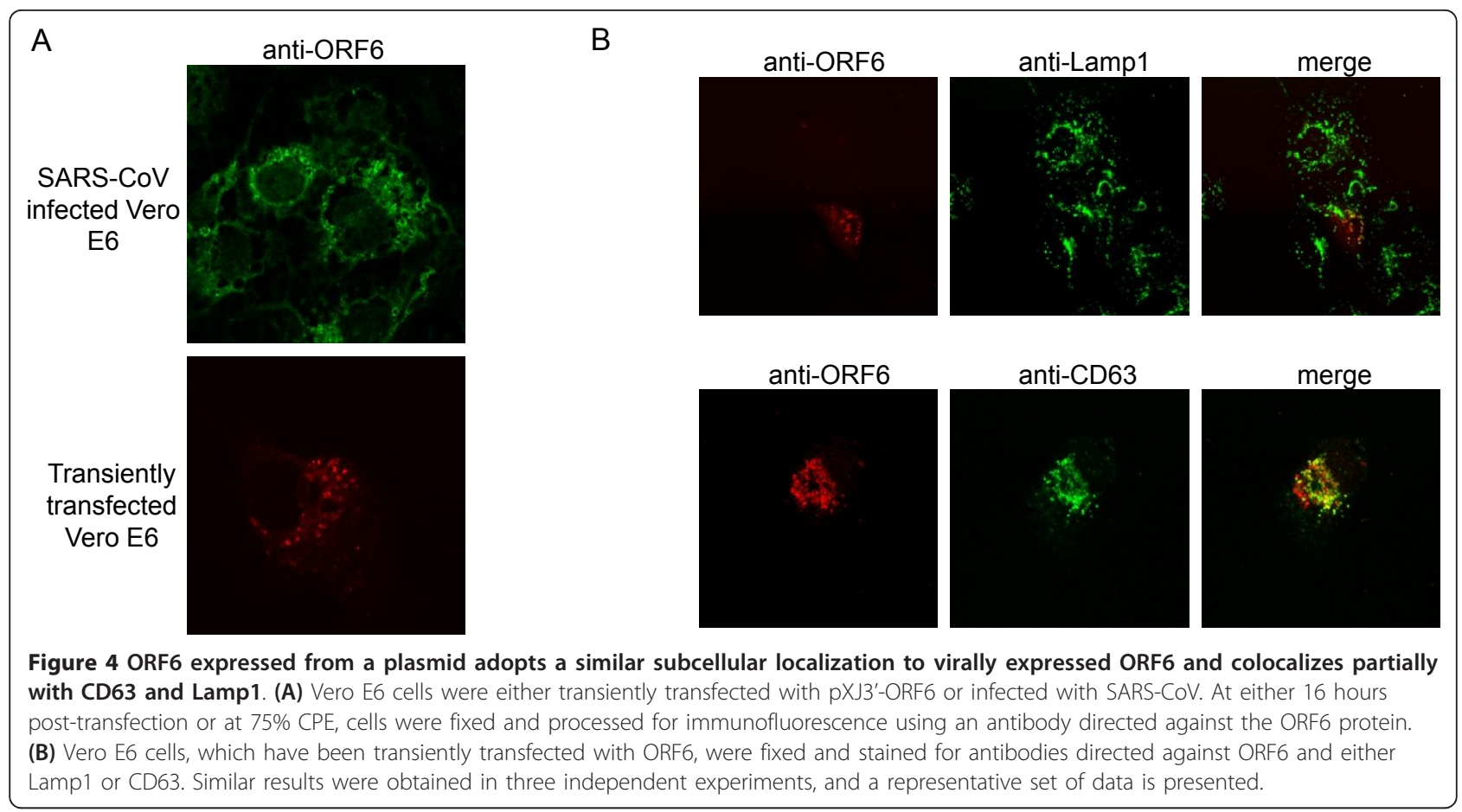

wildtype ORF6, ORF6A49-52 or ORF6A53-56. It was observed that both mutants showed increased levels of expression compared to wildtype ORF6, and this observation was reproducible (data not shown). The effect of ORF6A49-52 mutant on the expression of the nsp 8 gene was similar to wildtype ORF6 (Figure 5B). On the other hand, the nsp8 expression in the presence of ORF6A5356 was higher than that in the presence of wildtype ORF6 (Figure 5B), which suggest that ORF6A53-56 is less efficient in suppressing the expression of co-transfected myc-nsp8.

As the difference between wildtype ORF6 and ORF6A53-56 was subtle, a more quantitative approach to assay $n s p 8$ expression was deemed necessary. Hence, Vero E6 cells were transfected in the same manner and instead RNA was extracted and reverse transcribed and subjected to SYBR Green QPCR using primers for $n s p 8$. GAPDH was used as a housekeeping gene in order to normalize the expression levels seen. When pXJmycnsp8 was co-transfected with $1 \mu \mathrm{g}$ of pXJ3'-ORF6, ORF6A49-52 or ORF6A53-56, the levels of $n s p 8$ mRNA were similar (Figure $5 \mathrm{C}$ ). However, at $2 \mu \mathrm{g}$, the levels of $n s p 8$ mRNA for both ORF6 mutants were slightly higher than wild-type ORF6 (Figure 5C). In order to determine if these differences are statistically significant, 5 independent experiments were performed, and the results were then used to perform a t-test measuring the significance of the difference between $n s p 8$ expression levels when either $2 \mu \mathrm{g}$ of wildtype ORF6 or $2 \mu \mathrm{g}$ of each mutant were used. The difference in $n s p 8$ expression when co- transfected with either wildtype ORF6 and ORF6A49-52 is not statistically significant (a two-tailed p-value of $0.08)$. On the other hand, the difference in $n s p 8$ expression when co-transfected with either wildtype ORF6 and ORF6A53-56 is statistically significant (a two-tailed pvalue of 0.01 ). This indicated that the reduction in the suppression of the expression of co-transfected mycnsp 8 by ORF6A53-56 was significant, and therefore that the putative diacidic motif defined by amino acids 53-56 has a role to play in this ability of the ORF6 protein. The ORF6A49-52 mutant did not show as high a level of significance.

\section{Alanine substitutions at amino acids 53-56 alter the subcellular localization of ORF6}

Next, the subcellular localization of ORF6A49-52 and ORF6A53-56 was compared to wildtype ORF6. Vero E6 cells were transiently transfected with either pXJ3'ORF6, pXJ3'-ORF6A49-52 or pXJ3'-ORF6A53-56 and subjected to immunofluoresence analysis. It was observed that the localization of ORF6A49-52 was somewhat similar to wild-type ORF6 with main expression in distinctive vesicles (Figure 6). However, slightly more ORF6A49-52 than wildtype ORF6 was found to spread diffusely throughout the cytoplasm. The ORF6A53-56 also has some cytoplasmic staining but it was also observed that this mutant protein localized to vesicles that were clustered into groups of 3-5 vesicles to form large aggregates. Careful examination of the localization of the wildtype protein did not yield similar 


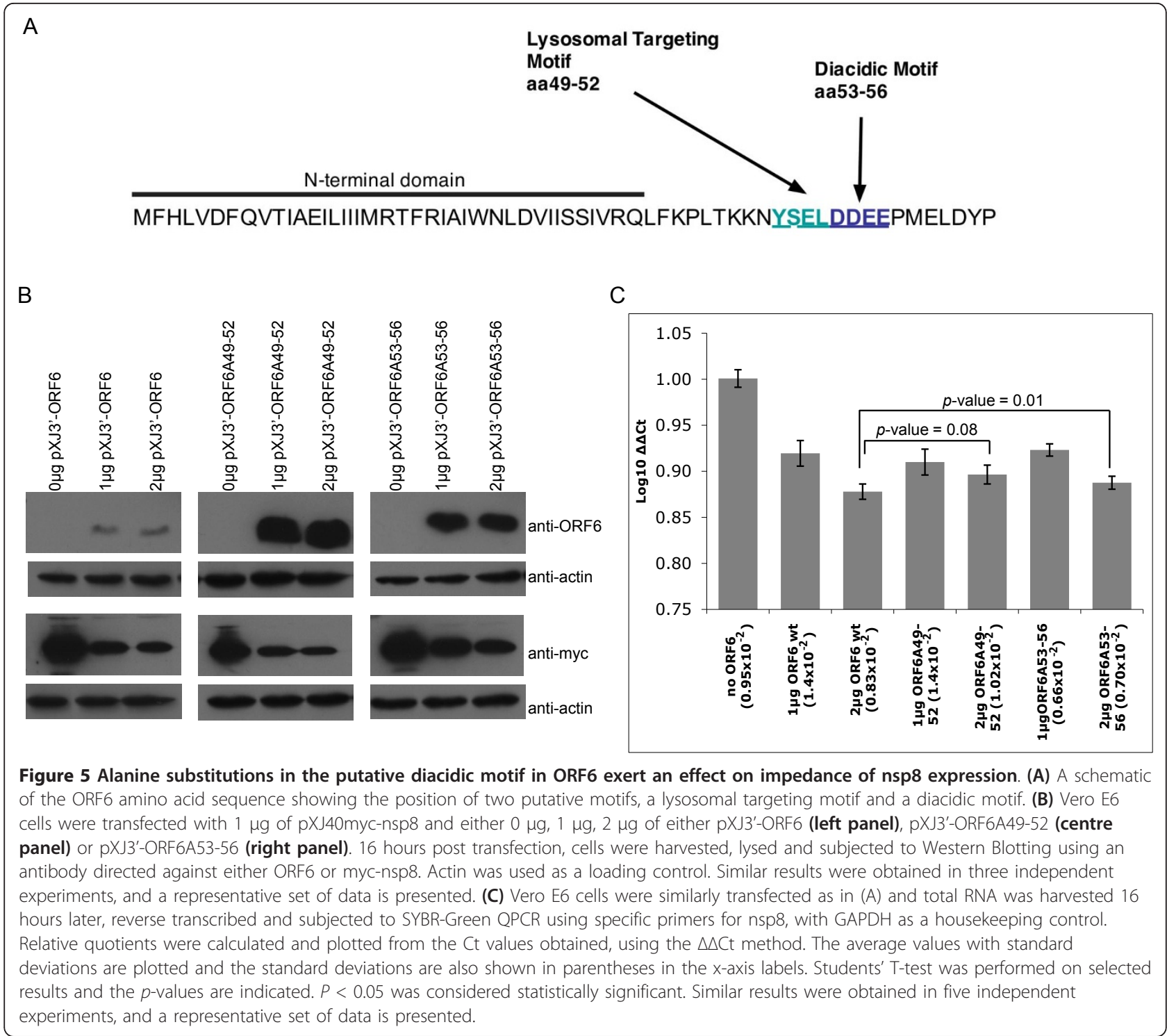

observations of clusters. This indicated that the putative diacidic motif from amino acids 53-56, in addition to being involved in the suppression of the expression of co-transfected myc-nsp8, is also involved in the subcellular localization of the ORF6 protein and therefore these 2 phenomena may be linked.

\section{Discussion}

Work done by several groups have shown that the ORF6 protein interacts with the nsp8 primase and localizes to a lysosomal compartment (5), impedes the nuclear translocation of STAT1 and other proteins specifically governed by the importin- $\beta$ superfamily (9), is able to enhance replication of both SARS-CoV (6) and MHVJHM (20), induces membrane rearrangements by means of its C-terminus (10) and induces apoptosis via a JNK- dependent mechanism (21). Through the use of alanine substitutions, many of these functions have been attributed to specific regions of the ORF6 protein.

In this study, we have employed the use of transiently transfected mammalian expression plasmids encoding for ORF6 to study its function. We have shown that ORF6 expressed in our system is functionally similar to that shown by others, by means of 2 assays. Firstly, the ORF6 expressed in our system is able to suppress the expression of a co-transfected CMV promoter-driven plasmid expressing either the nsp8 protein or GST. Secondly, the localization of ORF6 expressed via transient transfection was compared to the localization of virally expressed ORF6, and both display similar subcellular localization as well as colocalization to the Lamp1 marker. 


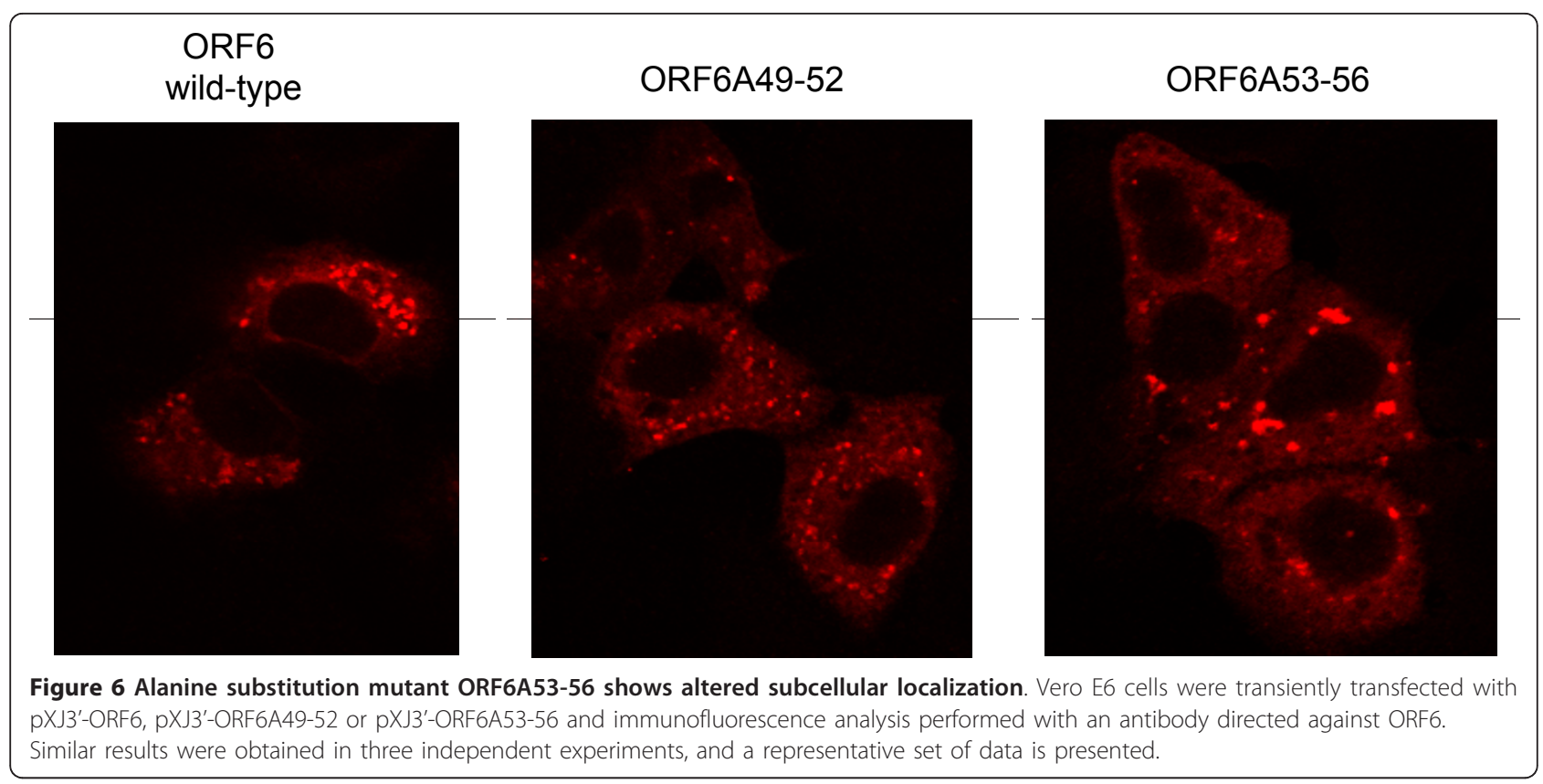

Using a bioinformatics approach to defining regions of interest in the ORF6 gene, we have identified 2 regions of interest, a putative lysosomal targeting motif YSEL between amino acids 49 to 52 and a putative diacidic motif DDEE between amino acids 53 to 56 (Figure 5A). Our results indicate that the putative diacidic motif is involved in both the suppression of expression of cotransfected expression constructs by ORF6 as well as its subcellular localization. While we were not able to completely restore the expression of co-transfected nsp8 in our system, the effect on nsp8 mRNA level between wildtype ORF6 and ORF6A53-56 was nonetheless found to be significantly different. However, the difference is small and suggests that the diacidic motif is largely dispensable for this function of ORF6. In contrast, the putative lysosomal targeting motif YSEL does not appear to contribute to these properties of ORF6 and this is consistent with a recent report that alanine substitution of the region from amino acids 49 to 53 fails to inhibit the ability of the protein to impede the nuclear translocation of STAT1 (9). Also, others have shown that the ORF6 protein induces membrane rearrangements and that the $\mathrm{C}$ terminus of the protein is important to this function. Our results show that the region from amino acids 53 to 56 is involved in the subcellular localization of the ORF6 protein, since alanine substitution of this region results in a clustered-vesicle phenotype that is not observed with wildtype ORF6. The reason for the difference in the subcellular localization of wild-type ORF6 and ORF6A53-56 mutant is not clear. One possibility is that the substitutions of four acidic residues with alanine residues might have reduced the solubility of ORF6. Alternatively, the substitutions may have disrupted the interaction of ORF6 with certain host proteins.

\section{Conclusions}

Taking these results together, the putative diacidic motif in ORF6 seems to be important for two properties of ORF6, namely its ability to suppress the expression of co-transfected expression constructs and its subcellular localization to vesicular structures. The implication of this is that the 2 functions may be inextricably linked. However, a cause-effect relationship has yet to be determined for these functions and in light of our findings, their relationship to the membrane rearrangements induced by the ORF6 protein should be examined in future studies. In addition, while the ability of ORF6 to suppress the expression of co-transfected expression constructs, was previously linked to the blockage of nuclear translocation (17), further experiments are needed to ascertain if amino acids 53-56 are involved in regulating the nuclear translocation process.

\section{Author details}

'Center for Microbiological Preparedness, Swedish Institute for Infectious Disease Control, SE-171 82 Solna, Sweden. ${ }^{2}$ Department of Microbiology, Tumor and Cell Biology, Karolinska Institutet, Nobels Väg 16, SE-17177 Stockholm, Sweden. ${ }^{3}$ Institute of Molecular and Cell Biology, 61 Biopolis Drive, A*STAR (Agency for Science, Technology and Research), Singapore 138673, Singapore. ${ }^{4}$ Department of Microbiology, Yong Loo Lin School of Medicine, National University Health System, National University of Singapore, MD4, 5 Science Drive 2, Singapore 117597, Singapore.

\section{Authors' contributions}

VG carried out the experiments and drafted the manuscript. AM and YJT participated in the design of the study and coordination and revised the manuscript. All authors read and approved the final manuscript. 


\section{Competing interests}

The authors declare that they have no competing interests

Received: 24 August 2011 Accepted: 25 October 2011

Published: 25 October 2011

\section{References}

1. Tan Y-J, Lim SG, Hong W: Understanding the accessory viral proteins unique to the severe acute respiratory syndrome (SARS) coronavirus. Antiviral Research 2006, 72(2):78-88.

2. Narayanan K, Huang C, Makino S: SARS coronavirus accessory proteins. Virus Research 2008, 133(1):113-21.

3. Yount B, Roberts RS, Sims AC, Deming D, Frieman MB, Sparks J, et al: Severe Acute Respiratory Syndrome Coronavirus Group-Specific Open Reading Frames Encode Nonessential Functions for Replication in Cell Cultures and Mice. Journal of Virology 2005, 79(23):14909-14922.

4. Netland J, Ferraro D, Pewe L, Olivares H, Gallagher T, Perlman S: Enhancement of murine coronavirus replication by severe acute respiratory syndrome coronavirus protein 6 requires the $\mathrm{N}$-terminal hydrophobic region but not C-terminal sorting motifs. Journal of Virology 2007, 81(20):11520-5

5. Kumar P, Gunalan V, Liu B, Chow VTK, Druce J, Birch C, et al: The nonstructural protein 8 (nsp8) of the SARS coronavirus interacts with its ORF6 accessory protein. Virology 2007, 366(2):293-303.

6. Zhao J, Falcón A, Zhou H, Netland J, Enjuanes L, Pérez Breña P, et al: Severe acute respiratory syndrome coronavirus protein 6 is required for optimal replication. Journal of Virology 2009, 83(5):2368-7.

7. Geng H, Liu Y-M, Chan W-S, Lo AW-I, Au DM-Y, Waye MM-Y, et al: The putative protein 6 of the severe acute respiratory syndrome-associated coronavirus: expression and functional characterization. FEBS Letters 2005, 579(30):6763-8.

8. Kopecky-Bromberg SA, Martínez-Sobrido L, Frieman M, Baric RA, Palese P: Severe acute respiratory syndrome coronavirus open reading frame (ORF) 3b, ORF 6, and nucleocapsid proteins function as interferon antagonists. Journal of Virology 2007, 81(2):548-57.

9. Frieman $M$, Yount B, Heise M, Kopecky-Bromberg SA, Palese P, Baric RS: Severe acute respiratory syndrome coronavirus ORF6 antagonizes STAT1 function by sequestering nuclear import factors on the rough endoplasmic reticulum/Golgi membrane. Journal of Virology 2007 , 81(18):9812-24.

10. Zhou H, Ferraro D, Zhao J, Hussain S, Shao J, Trujillo J, et al: The N-terminal region of severe acute respiratory syndrome coronavirus protein 6 induces membrane rearrangement and enhances virus replication. Journal of Virology 2010, 84(7):3542-51.

11. Miranda-Saksena M, Boadle RA, Aggarwal A, Tijono B, Rixon FJ, Diefenbach RJ, et al: Herpes simplex virus utilizes the large secretory vesicle pathway for anterograde transport of tegument and envelope proteins and for viral exocytosis from growth cones of human fetal axons. Journal of Virology 2009, 83(7):3187-99.

12. Chambers R, Takimoto T: Trafficking of Sendai virus nucleocapsids is mediated by intracellular vesicles. PLoS One 2010, 5(6):e10994

13. Sims AC, Ostermann J, Denison MR: Mouse hepatitis virus replicase proteins associate with two distinct populations of intracellular membranes. Journal of Virology 2000, 74(12):5647-54.

14. Snijder EJ, van der Meer $Y$, Zevenhoven-Dobbe J, Onderwater JJM, van der Meulen J, Koerten HK, et al: Ultrastructure and origin of membrane vesicles associated with the severe acute respiratory syndrome coronavirus replication complex. Journal of Virology 2006, 80(12):5927-40.

15. Knoops K, Kikkert M, Worm SHED, Zevenhoven-Dobbe JC, van der Meer $Y$, Koster AJ, et al: SARS-coronavirus replication is supported by a reticulovesicular network of modified endoplasmic reticulum. PLOS Biology 2008, 6(9):e226.

16. Reggiori F, Monastyrska I, Verheije MH, Cali T, Ulasli M, Bianchi S, Bernasconi R, de Haan CA, Molinari M: Coronaviruses Hijack the LC3-Ipositive EDEMosomes, ER-derived vesicles exporting short-lived ERAD regulators, for replication. Cell Host Microbe 2010, 7(6):500-8.

17. Hussain S, Perlman S, Gallagher TM: Severe acute respiratory syndrome coronavirus protein 6 accelerates murine hepatitis virus infections by more than one mechanism. Journal of Virology 2008, 82(14):7212-22.
18. Williams MA, Fukuda M: Accumulation of membrane glycoproteins in lysosomes requires a tyrosine residue at a particular position in the cytoplasmic tail. The Journal of Cell Biology 1990, 111(3):955-66.

19. Nishimura NA: Di-Acidic Signal Required for Selective Export from the Endoplasmic Reticulum. Science 1997, 277(5325):556-8.

20. Pewe L, Zhou H, Netland J, Tangudu C, Olivares H, Shi L, et al: A Severe Acute Respiratory Syndrome-Associated Coronavirus-Specific Protein Enhances Virulence of an Attenuated Murine Coronavirus. Journal of Virology 2005, 79(17):11335-42.

21. Ye Z, Wong CK, Li P, Xie Y: A SARS-CoV protein, ORF-6, induces caspase-3 mediated, ER stress and JNK-dependent apoptosis. Biochimica et Biophysica Acta 2008, 1780(12):1383-7.

doi:10.1186/1756-0500-4-446

Cite this article as: Gunalan et al:: A putative diacidic motif in the SARSCoV ORF6 protein influences its subcellular localization and suppression of expression of co-transfected expression constructs. BMC Research Notes 2011 4:446.

\section{Submit your next manuscript to BioMed Central and take full advantage of:}

- Convenient online submission

- Thorough peer review

- No space constraints or color figure charges

- Immediate publication on acceptance

- Inclusion in PubMed, CAS, Scopus and Google Scholar

- Research which is freely available for redistribution 